\title{
A Spectrum Sensing Scheme for Partially Polarized Waves over $\alpha-\mu$ Generalized Gamma Fading Channels
}

\author{
Mohamed A. Hankal, Islam A. Eshrah, and Hazim Tawfik \\ Electronics and Electrical Communications Engineering Department, Faculty of Engineering, Cairo University, Giza 12613, Egypt \\ Correspondence should be addressed to Mohamed A. Hankal; mohamedhankal@gmail.com
}

Received 9 November 2013; Accepted 19 December 2013; Published 9 February 2014

Academic Editors: N. Bouguila and C.-M. Kuo

Copyright (C) 2014 Mohamed A. Hankal et al. This is an open access article distributed under the Creative Commons Attribution License, which permits unrestricted use, distribution, and reproduction in any medium, provided the original work is properly cited.

\begin{abstract}
Schemes for spectrum holes sensing for cognitive radio based on the estimation of the Stokes parameters of monochromatic and quasimonochromatic polarized electromagnetic waves are developed. Statistical information that includes the variations of the polarization state in both cases (present and absent) of Primary User (PU) is accounted for. A detector based on the fluctuation of the Stokes parameters is analyzed, and its performance is compared with that of energy detectors, which use only the scalar amplitude information to sense the PU signal. The cooperative spectrum sensing based on the polarization in which the reporting channels are noisy will be investigated. The cluster technique is proposed to reduce the bit error probability due to channel impairment. A closed-form expression for the polarization detection is derived using $\alpha-\mu$ generalized fading model, which provides directly an expression for the special cases of Nakagami-m and Weibull models as well as their derivatives. These expressions are verified using simulation. The results show that the polarization spectrum sensing gives superior performance for a wide range of SNR over the conventional energy detection method.
\end{abstract}

\section{Introduction}

Cognitive radio (CR) technology has witnessed a growing interest over the past decade, as it promises more efficient use of the available spectrum [1,2]. A key stage in CR is spectrum sensing, in which the Secondary User (SU) must detect the presence of a Primary User (PU) in a certain channel, and thus, deems this part of the spectrum unused, and make the decision to share it. This entails a sequence of functions that the CR system should perform, such as power control [3] and spectrum management [4]. Several techniques were proposed to improve spectrum sensing such as energy detection [5], cyclostationary feature detection [6], sensing based on smart antennas [7, 8], and wideband spectrum sensing $[9,10]$. These techniques primarily make use of the amplitude, frequency, and phase information of the PU signal.

It is possible, however, to improve the spectrum sensing process by exploiting the polarization state of the signal. In radar systems [11], the polarization state was used to improve the detection capability of the system. The new polarization-dependent detection statistics, which use the power and relative phase of the two orthogonal polarization components was proposed to enhance radar detection in homogenous channels [12]. The radar detection performance was enhanced based on the polarization difference between the clutter and the target [11]. The sine of the relative phase between two orthogonally polarized received signals has been proposed and tested as detection statistic in radar systems [13]. New statistics use two orthogonal polarization component powers and their relative phase to enhance target detection [12]. They are thus fundamentally different from the well-known Marcum-Swerling envelope detector [14], which operates on only a single polarization component of the received power, and the pseudocoherent detector [13], which operates on only the relative phase of the polarization components.

Recent research on polarization detection in CR was concerned with completely polarized waves. A Virtual Polarization Detection (VPD) method based on the vector signal processing was presented for effective spectrum sensing of cognitive radios [15]. Polarization spectrum hole sensing was 
proposed for cognitive radio to optimize the received polarization at the SU in order to protect the PU from interference caused by the SU and to reduce the interference from PU to SU [16]. Optimal Polarization Reception (OPR) was proposed for CR to improve the SINR [17]. A new blind spectrum sensing method based on the polarization characteristic of the received signal, which is completely represented by the orientation of a polarization vector, was proposed [18], and a closed-form expression for the probability of false alarm and probability of detection under Additive White Gaussian Noise (AWGN) and Rayleigh-fading channels was derived. The fading and noisy nature of a wireless communication channel places a major challenge for spectrum sensing. Since sensing decisions based on a single SU measurements may be unreliable, the idea of collaborative spectrum sensing has attracted a lot of research interest [19]. However, the polarization state is changed with spectrum sensing time which was not considered in this work.

In this paper, the above constraint is addressed by proposing a polarization-based spectrum sensing scheme where a statistical model for the PU signal polarization parameters is adopted. This model takes into account the channel backscatter and the partial polarization nature of the PU signal, with the assumption that the channel experiences slow fading. Cooperative polarization spectrum sensing is proposed to mitigate the effects of fading and shadowing, which can seriously degrade the sensing performance. A cluster-based cooperation scheme is proposed to decrease bit error probability. All SUs are grouped into few clusters and one cluster head is set for each cluster to collect the sensing results, make cluster decisions, and forward measurements to the central unit. Thus the bit error probability will be reduced greatly because most of SUs will be closer to the cluster heads than to the central unit. Analytical results show that significant improvement can be achieved with our proposed method.

The rest of this paper is organized as follows. In Section 2, the method of characterizing the signal polarization is provided. The spectrum sensing models are developed in Section 3. Section 4 describes the cooperative spectrum sensing mechanism and the cluster technique of the cooperative sensing is shown in Section 5. Simulation results are illustrated in Section 6, followed by the conclusion in Section 7.

\section{The Method of Characterizing the Signal Polarization}

The polarization of a monochromatic plane wave is completely specified by constant amplitude and relative phase of the two orthogonal electric-field components.

In a cognitive radio system, the SU uses orthogonally dual polarized antennas to detect the PU signal $\vec{E}$, which can be completely described in vector form as

$$
\vec{E}=\left[\begin{array}{l}
E_{h} \\
E_{v}
\end{array}\right]=\left[\begin{array}{c}
E_{h}^{i}+j E_{h}^{q} \\
E_{v}^{i}+j E_{v}^{q}
\end{array}\right],
$$

where $i$ and $q$ indicate the in-phase and quadrate phase components, respectively. Typically, the polarization of a monochromatic wave is determined by the Jones vector $\vec{J}$ of the signal $\vec{E}$ defined as [20]

$$
\vec{J}=\left[\begin{array}{c}
\cos \zeta \\
e^{j \theta} \sin \zeta
\end{array}\right],
$$

where $\zeta=\tan ^{-1}\left(\left|E_{v}\right| /\left|E_{h}\right|\right)$ and $\theta=\tan ^{-1}\left(\left|E_{v}^{q}\right| /\left|E_{v}^{i}\right|\right)-\tan ^{-1}$ $\left(\left|E_{h}^{q}\right| /\left|E_{h}^{i}\right|\right)$.

Alternatively, the polarization is determined by the geometrical parameters, namely the ellipticity angle $\tau$, and orientation angle $\phi$, which can be uniquely represented by a point on the Poincare sphere. The field component parameters $\zeta$ and $\theta$, and the geometrical parameters $\tau$ and $\phi$, are described in the following set of equations [21]

$$
\begin{gathered}
\sin (2 \zeta) \cos (\theta)=\sin (2 \phi) \cos (2 \tau), \\
\sin (2 \zeta) \sin (\theta)=\sin (2 \tau), \\
\cos (2 \zeta)=\cos (2 \phi) \cos (2 \tau) .
\end{gathered}
$$

However, if the amplitudes and phases encounter slow fluctuations with time, which is the case if the polarization of primary signal suffers from either noise or fading, the components of the Jones vector are said to be quasimonochromatic or narrowband. The polarization of a quasimonochromatic wave is quantified using an average polarization state vector, which may be defined in terms of four measurable components; namely, $\vec{X}=\left[x_{0} x_{1} x_{2} x_{3}\right]^{T}$. These components are known as the Stokes parameters (SPs) and are given by [12]

$$
\begin{gathered}
x_{0}=\frac{1}{N} \sum_{n=1}^{N}\left[\left(E_{h}^{i}\left(n T_{s}\right)\right)^{2}+\left(E_{h}^{q}\left(n T_{s}\right)\right)^{2}\right. \\
\left.+\left(E_{v}^{i}\left(n T_{s}\right)\right)^{2}+\left(E_{v}^{q}\left(n T_{s}\right)\right)^{2}\right], \\
x_{1}=\frac{1}{N} \sum_{n=1}^{N}\left[\left(E_{h}^{i}\left(n T_{s}\right)\right)^{2}+\left(E_{h}^{q}\left(n T_{s}\right)\right)^{2}\right. \\
\left.-\left(E_{v}^{i}\left(n T_{s}\right)\right)^{2}-\left(E_{v}^{q}\left(n T_{s}\right)\right)^{2}\right], \\
x_{2}=\frac{2}{N} \sum_{n=1}^{N}\left[E_{h}^{i}\left(n T_{s}\right) E_{v}^{i}\left(n T_{s}\right)+E_{h}^{q}\left(n T_{s}\right) E_{v}^{q}\left(n T_{s}\right)\right], \\
x_{3}=\frac{2}{N} \sum_{n=1}^{N}\left[E_{h}^{i}\left(n T_{s}\right) E_{v}^{q}\left(n T_{s}\right)-E_{v}^{i}\left(n T_{s}\right) E_{h}^{q}\left(n T_{s}\right)\right],
\end{gathered}
$$

where $T_{s}$ is the sampling time, and $N$ is the number of samples which is restricted by the bandwidth of a narrowband filter. $x_{0}, x_{1}, x_{2}$, and $x_{3}$ are physically recognizable quantities as follows.

(1) $x_{0}$ is the sum of the power in the $h$ and $v$ electric-field components and thus represents the total power of the received signal.

(2) $x_{1}$ is the difference between the power in the $h$ and $v$ electric-field components. 
(3) $x_{2}$ is the difference between the power in the two orthogonal electric-field components whose axes are rotated $45^{\circ}$ relative to the $h$ and $v$ axes.

(4) $x_{3}$ is the difference between the right-hand and the left-hand circularly polarized power.

The component $x_{0}$ satisfies the relation

$$
x_{0} \geq x_{1}+x_{2}+x_{3} .
$$

Alternatively, the average polarization state can be obtained from the coherence matrix $\mathbf{C}$ of the received field, such that

$$
\mathbf{C}=\left\langle\left[\begin{array}{cc}
E_{h} E_{h}^{*} & E_{h} E_{v}^{*} \\
E_{v} E_{h}^{*} & E_{v} E_{v}^{*}
\end{array}\right]\right\rangle,
$$

where $\langle\cdot\rangle$ denotes averaging over $N T_{s}$ and $*$ represents the complex conjugate. The coherence matrix $\mathbf{C}$ is a linear combination of the SPs such that:

$$
\mathbf{C}=x_{0} \mathbf{F}_{0}+x_{1} \mathbf{F}_{1}+x_{2} \mathbf{F}_{2}+x_{3} \mathbf{F}_{3} \text {, }
$$

where $\mathbf{F}_{0}=0.5\left[\begin{array}{ll}1 & 0 \\ 0 & 1\end{array}\right], \mathbf{F}_{1}=0.5\left[\begin{array}{cc}1 & 0 \\ 0 & -1\end{array}\right], \mathbf{F}_{2}=0.5\left[\begin{array}{ll}0 & 1 \\ 1 & 0\end{array}\right]$, and $\mathbf{F}_{3}=0.5\left[\begin{array}{cc}0 & 1 \\ -1 & 0\end{array}\right]$.

The relationship of the SPs to the geometrical parameters $(\phi, \tau)$ is shown in the following set of equations:

$$
\begin{gathered}
x_{1}=\cos (2 \phi) \cos (2 \tau), \\
x_{2}=\sin (2 \phi) \cos (2 \tau), \\
x_{3}=\sin (2 \tau) .
\end{gathered}
$$

Thus far, the orientation of a polarization vector on the unit Poincare sphere can completely represent the polarization state of the received signal.

\section{Spectrum Sensing Based on the Polarization}

Spectrum sensing is essentially a binary hypothesis testing problem, which indicates the PU's absence or presence, respectively, such that

$$
Y(t)=\left\{\begin{array}{cc}
h E(t)+W(t) & H_{1} \\
W(t) & H_{0}
\end{array}\right\}
$$

where $Y(t)$ is the observed signal at the CR, $E(t)$ is the PU signal, $W(t)$ is the zero mean Gaussian random process with identical autocorrelation and power spectral density $N_{0}$ Watts $/ \mathrm{Hz}$, and $h$ is the amplitude gain of the channel having mean-square value $\Omega=\bar{h}^{2}$ and Probability Density Function (PDF) $f_{h}(h)$. The received instantaneous signal power is modulated by $h^{2}$ and consequently the instantaneous Signalto-Noise Ratio (SNR) can be expressed as $\gamma=h^{2}\left(E s / N_{0}\right)$ with an average $\bar{\gamma}=\Omega\left(E s / N_{0}\right)$ where $E_{s}$ is the signal energy accumulated over the observation period.

In this paper, we propose two schemes which use the degree of polarization and the axial ratio to detect the PU signal.
3.1. Spectrum Sensing Based on the Degree of Polarization. The degree of polarization $(D)$ is a quantity used to describe the polarized portion of an electromagnetic wave. A perfectly polarized wave has $D$ equal to 1 , whereas an unpolarized wave has $D$ equal to 0 . A wave, which is partially polarized, can therefore be represented by superposition of a polarized and unpolarized component. This implies a $D$ somewhere in between 0 and 1 . Alternatively, the degree of polarization $D$ is defined as the ratio of the polarized power to the total power in the wave; that is,

$$
D=\frac{\sqrt{x_{1}^{2}+x_{2}^{2}+x_{3}^{2}}}{x_{0}} .
$$

The estimate of the ratio of the polarized power to the total power in the received signal is used as detection statistics in radar systems [12]. Figure 1 depicts a block diagram of the proposed spectrum sensing system where $D_{S}=D$. There are two orthogonal antennas, which detect the horizontal and vertical components $E_{h}$ and $E_{v}$ of the signal $E$, respectively. The Stokes vector estimation block delivers the Stokes vector $\vec{X}$ to the polarization degree estimator, which, in turn, produces the detection statistic $D$. This statistic serves as the input to the threshold detector to decide whether the PU is present or not.

The distribution that governs the statistic $D$ can be used to estimate the probability of detection and the probability of false alarm. Here, the in-phase and quadrature components of the quasimonochromatic wave are assumed to be zeromean Gaussian random processes. The estimation of the SP and the elements of the sample correlation random process is equivalent, which is called Wishart distribution [22]. Thus, the probability density function of the detection statistic $D$ is given by [12]

$$
\begin{aligned}
P(x)= & \frac{2 \Gamma(N-1 / 2)}{\sqrt{\pi} \Gamma(N-1)} \frac{\left[1-D_{\infty}^{2}\right]^{N}\left[1-x^{2}\right]^{N-2}}{\left[1-D_{\infty}^{2} x^{2}\right]^{2 N-1}} \\
& \times \sum_{k=1}^{N} \frac{\Gamma(2 N) D_{s}^{2 k-2} x^{2 k}}{\Gamma(2 k) \Gamma(2 N-2 k+1)},
\end{aligned}
$$

where $D_{\infty}$ and $\left\{s_{0}, s_{1}, s_{2}, s_{3}\right\}$ are the actual degree of polarization, and the actual Stokes vector components, respectively. They are obtained in the limit as the number of independent samples approaches infinity. For a fixed threshold $\lambda$, the conditional probability of false alarm $P_{f}$ and detection $P_{d}$ can be expressed as [12]

$$
\begin{gathered}
P_{f}=\frac{1}{2} I_{1-\lambda^{2}}\left(N-1, \frac{3}{2}\right), \\
P_{d}=1-A\left[1-\left(\frac{\gamma}{1+\gamma}\right)^{2}\right]^{N} \\
\times \sum_{k=1}^{N} B_{k}\left(\frac{\gamma}{1+\gamma}\right)^{2 k-2} \sum_{n=0}^{\infty} C_{k, n}\left(\frac{\gamma}{1+\gamma}\right)^{2 n},
\end{gathered}
$$

where $\gamma$ is the Signal-to-Noise Ratio (SNR), $I_{1-\lambda^{2}}(n+1 / 2, N)$ is the incomplete beta function, $A=2 \Gamma(N+1 / 2) / \sqrt{\pi}$, 


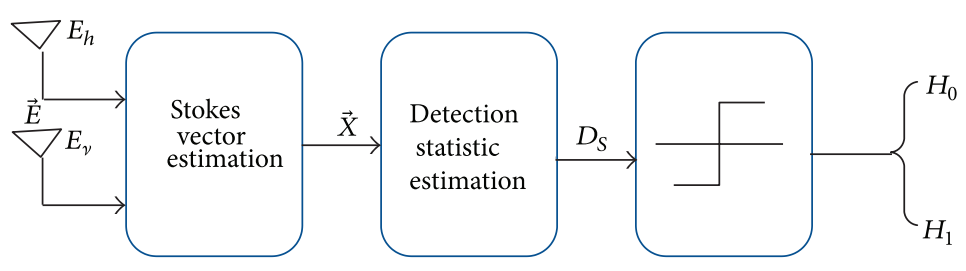

FIGURE 1: The proposed spectrum sensing system model.

$B_{k}=1 / \Gamma(2 k) \Gamma(2 N-2 k+1)$, and $C_{k, n}=\Gamma(2 N+n-1) \Gamma(n+k-$ $1 / 2) /(\Gamma(n+1) \Gamma(N+n+k-1 / 2)) I_{\lambda^{2}}(k+n+1 / 2, N-1)$. The SNR $\gamma$ can be affected by fading that in turn affects $P_{d}$ and hence in order to incorporate its influence, $P_{d}$ must be averaged over all possible values of $\gamma$ according to

$$
\overline{P_{d}}=\int_{0}^{\infty} P_{d}(\gamma) f_{\gamma}(\gamma) d \gamma
$$

where $f(\cdot)$ represents the PDF of the channel. For a fading signal with envelope $h$, an arbitrary parameter $\alpha>0$, and a $\alpha$-root mean value $\bar{h}=\sqrt[\alpha]{E\left(h^{\alpha}\right)}$, the $\alpha-\mu$ PDF $f_{h}(h)$ is given by [23]

$$
f_{h}(h)=\frac{\alpha \mu^{\mu} h^{\alpha \mu-1} e^{-\mu(h / \bar{h})^{\alpha}}}{\Gamma(\mu) \bar{h}^{-\alpha \mu}},
$$

where $\mu=\mathscr{E}^{2}\left(h^{\alpha}\right) / \mathscr{V}\left(h^{\alpha}\right)$ and $\mathscr{E}(\cdot)$ and $\mathscr{V}(\cdot)$ are the expectation and variance operators, respectively [23]. The PDF of the SNR is obtained by a change of variables as shown in [24]

$$
f_{\gamma}(\gamma)=\frac{(\alpha / 2) \mu^{\mu} \gamma^{\alpha \mu / 2-1}}{\Gamma(\mu) \bar{\gamma}^{\alpha \mu / 2}} e^{-\mu(\gamma / \bar{\gamma})^{\alpha / 2}} .
$$

By substituting (15) in (13), $\overline{P_{d}}$ can be written as

$$
\bar{P}_{d}=1-A^{\prime} \sum_{k=1}^{N} B_{k} \sum_{n=0}^{\infty} C_{k, n} \int_{0}^{\infty} g_{n}(\gamma) d \gamma
$$

where

$$
\begin{aligned}
& g_{n}(\gamma)= {\left[1-\left(\frac{\gamma}{1+\gamma}\right)^{2}\right]^{N} } \\
& \times\left(\frac{\gamma}{1+\gamma}\right)^{2 n+2 k-2} \gamma^{\alpha / 2 \mu-1} e^{-\mu(\gamma / \bar{\gamma})^{\alpha / 2}}, \\
& A^{\prime}=\frac{\alpha / 2 \mu^{\mu}}{\Gamma(\mu) \bar{\gamma}^{-\alpha \mu / 2}} A .
\end{aligned}
$$

Using [25], one can get the average probability of detection $\bar{P}_{d}$ as follows:

$$
\begin{aligned}
\bar{P}_{d}= & 1-\sum_{k=1}^{N} B_{k} \sum_{n=0}^{\infty} C_{k, n} \sum_{r=0}^{N}\left(\begin{array}{c}
N \\
r
\end{array}\right)(-1)^{r} \\
& \times\left\{\begin{array}{cc}
A(v, \mu, z) & \alpha=1 \\
\Gamma(v+\mu) U(v, 1-\mu, z) & \alpha=2 \\
\frac{(z / k)^{v} l^{\mu+1 / 2}}{\sqrt{2 \pi}} G_{k+l, k}^{k, k+l} & \\
\times\left[\frac{2}{z^{k}} \mid \begin{array}{c}
\Delta(l,-\mu), \Delta(k, v+1) \\
\Delta(k, 0)
\end{array}\right] & \alpha \neq 1,2
\end{array}\right\},
\end{aligned}
$$

where

$$
\begin{aligned}
A(v, \mu, z)= & \frac{1}{\Gamma(v)} \\
& \times\left[{ }_{1} F_{2}\left(v ; \frac{1}{2}-\frac{\mu}{2}, 1-\frac{\mu}{2} ;-\frac{z}{4}\right) \Gamma(\mu) \Gamma(v)\right] \\
& \times{ }_{1} F_{2}\left(v+\frac{\mu}{2} ; \frac{1}{2}, \frac{\mu}{2}+1 ;-\frac{z}{4}\right) \frac{z^{\mu / 2}}{2} \\
& \times \Gamma\left(-\frac{\mu}{2}\right) \Gamma\left(v+\frac{\mu}{2}\right) \\
& -{ }_{1} F_{2}\left(v+\frac{\mu}{2}+\frac{1}{2} ; \frac{3}{2}, \frac{\mu}{2}+\frac{3}{2} ;-\frac{z}{4}\right), \\
& \quad v=2\left(N+n+k-\frac{\mu}{\bar{\gamma}},\right. \\
& { }_{1} F_{2}\left(v ; \frac{1}{2}-\frac{\mu}{2}, 1-\frac{\mu}{2} ;-\frac{z}{4}\right)
\end{aligned}
$$

is the Hypergeometric function, $G(\cdot,:)$ is the Meijer $G$ function, and $U$ is the Hypergeometric $U$ function [26].

3.2. Spectrum Sensing Based on the Axial Ratio of a Polarization Ellipse. The polarized portion of the PU signal represents a net polarization ellipse traced by the electric field vector as a function of time. The ellipse has a magnitude $(R)$ such that

$$
R=\left|\frac{x_{3}}{x_{0}}\right|
$$

The ellipticity is the ratio of the minor to the major axis of the corresponding electric field polarization ellipse and varies 
from 0 for linearly polarized wave to 1 for circularly polarized wave. The polarization ellipse is alternatively described by its eccentricity, which is zero for a circularly polarized wave, and increases as the ellipse becomes thinner. It then becomes one for a linearly polarized wave. Alternatively $R$ is defined as the ratio of the polarized power to the total power in the wave.

Figure 1 depicts a block diagram of the proposed spectrum sensing system, where $D_{S}=R$. This statistic serves as the input to the threshold detector to decide whether the PU is present or not. The distribution that governs the statistic $R$ is to be determined to estimate the probability of detection and the probability of false alarm [12], which is given by

$$
\begin{aligned}
& P_{R}(x) \\
& \quad=\frac{\Gamma(N+1 / 2)}{\sqrt{\pi} \Gamma(N)}\left[1-D_{\infty}^{2}\right]^{2}\left[1-x^{2}\right]^{N-1} \\
& \quad \times\left\{\frac{\left[1-\left(s_{3} x / s_{0}\right)\right]}{\left[\left(1-\left(s_{3} x / s_{0}\right)\right)^{2}-\left(s_{1}^{2}+s_{2}^{2} / s_{0}^{2}\right)\left(1-x^{2}\right)^{2}\right]^{N+1 / 2}}\right\} \\
& \quad+\left\{\frac{\left[1+s_{3} x / s_{0}\right]}{\left[\left(1+s_{3} x / s_{0}\right)^{2}+\left(\left(s_{1}^{2}+s_{2}^{2}\right) / s_{0}^{2}\right)\left(1-x^{2}\right)^{2}\right]^{N+1 / 2}}\right\} .
\end{aligned}
$$

For a fixed threshold $\lambda$, the conditional probability of false alarm $P_{f}$ can be expressed as

$$
P_{f}=\frac{1}{2} I_{1-\lambda^{2}}\left(N, \frac{1}{2}\right),
$$

and the probability of detection $P_{d}$ can be given by

$$
\begin{aligned}
P_{d}= & 1-\left[1-\left(\frac{\gamma}{1+\gamma}\right)^{2}\right]^{N} \\
& \times \sum_{n=0}^{\infty} \frac{\Gamma(N+n)}{\Gamma(n+1) \Gamma(N)} I_{\lambda^{2}}\left(n+\frac{1}{2}, N\right)\left(\frac{\gamma}{1+\gamma}\right)^{2 n} .
\end{aligned}
$$

$P_{d}$ must be averaged over all possible values of $\gamma$ as follows:

$$
\overline{P_{d}}=\int_{0}^{\infty} p_{d}(\gamma) f_{\gamma}(\gamma) d \gamma
$$

where $f_{\gamma}(\gamma)$ represents the $\alpha-\mu$ probability density function. Following a similar approach to that of Section 3.1 and by substituting (15) in (24), $\overline{P_{d}}$ can be written as

$$
\bar{P}_{d}=1-k \sum_{n=0}^{\infty} \frac{\Gamma(N+n)}{\Gamma(n+1) \Gamma(N)} I_{\lambda^{2}}\left(n+\frac{1}{2}, N\right) \int_{0}^{\infty} g_{n}(\gamma) d \gamma
$$

where

$$
\begin{gathered}
g_{n}(\gamma)=\left[1-\left(\frac{\gamma}{1+\gamma}\right)^{2}\right]^{N}\left(\frac{\gamma}{1+\gamma}\right)^{2 n} \gamma^{\alpha / 2 \mu-1} e^{-\mu(\gamma / \bar{\gamma})^{\alpha / 2}}, \\
k=\frac{\alpha \mu^{\mu}}{2 \Gamma(\mu) \bar{\gamma}^{\alpha \mu / 2}} .
\end{gathered}
$$

Using binomial theorem and [25], $\bar{P}_{d}$ will be given by

$$
\begin{aligned}
\bar{P}_{d}= & 1-\sum_{n=0}^{\infty} \frac{\Gamma(N+n)}{\Gamma(n+1) \Gamma(N)} \\
& \times I_{Y_{\text {th }}}\left(n+\frac{1}{2}, N\right) \sum_{r=0}^{N}\left(\begin{array}{c}
N \\
r
\end{array}\right)(-1)^{r} \\
& \times\left\{\begin{array}{cc}
A(v, \mu, z) & \alpha=1 \\
\Gamma(v+\mu) U(v, 1-\mu, z) & \alpha=2 \\
\frac{(z / k)^{v} l^{\mu+(1 / 2)}}{\sqrt{2 \pi}} G_{k+l, k}^{k, k+l} & \\
\times\left[\frac{2}{z^{k}} \mid \begin{array}{c}
\Delta(l,-\mu), \Delta(k, v+1) \\
\Delta(k, 0)
\end{array}\right] & \alpha \neq 1,2
\end{array}\right\} .
\end{aligned}
$$

It is worth mentioning that the restriction for this scheme is that the polarization information of the primary signal must be known a priori. If the PU signal is linearly polarized, then $R$ will be close to 0 for high SNR, and if the PU signal is circularly polarized, then $R$ will be close to 1 for high SNR.

\section{Spectrum Sensing Based on Cooperative Polarization Detection}

The fading and noisy nature of a wireless communication channel places a major challenge on the accuracy of spectrum sensing. Sensing decisions that is based on measurements of a single SU may be unreliable. Cooperative spectrum sensing is one possible solution to overcome this unreliability. In Figure 2, a number of SUs, which are distributed in different locations independently, can detect the PU and make the decision whether the signal exists or not. According to the information received from various SUs, the central unit makes the final decision based on some rules. A general fusion rule is when a final decision of 1 is taken when $m$ out-of- $N$ SUs report 1 . When $m=1$, the $m$-out-of $-N$ rule is equivalent to the OR rule. When $m=N$, the decision rule becomes the AND rule. By selecting different values of $m$, different detection performances are obtained.

Two cases are considered, namely, cooperative spectrum sensing with perfect and imperfect reporting channels.

4.1. Perfect Reporting Channels. If the channels between each SU and the central unit are noise free, then the overall probability of false alarm $Q_{F}$ and the overall probability of detection $Q_{D}$ of the cooperative spectrum sensing for $m$-outof $-N$ rule of the cooperative spectrum sensing are given by [27]

$$
\begin{aligned}
& Q_{F}=\sum_{i=m}^{N}\left(\begin{array}{c}
N \\
i
\end{array}\right)\left(P_{f, i}\right)^{i}\left(1-P_{f, i}\right)^{N-i}, \\
& Q_{D}=\sum_{i=m}^{N}\left(\begin{array}{c}
N \\
i
\end{array}\right)\left(P_{d, i}\right)^{i}\left(1-P_{d, i}\right)^{N-i},
\end{aligned}
$$

where the $P_{f, i}$ and $P_{d, i}$ are the probabilities of false alarm and detection of the degree of polarization and axial ratio as derived in (14), (18), (22), and (27), respectively. 


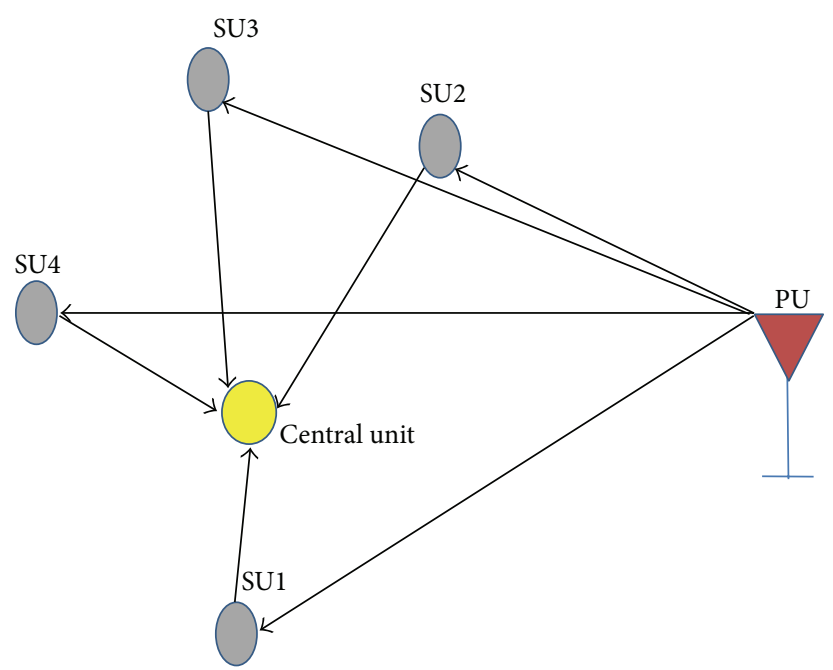

FIGURE 2: System model of the cooperative network.

4.2. Imperfect Reporting Channels. In practical systems, the reporting channels between the SUs and the central unit will experience fading. This, in turn, will degrade transmission reliability of the sensing results that are reported from the SUs to the central unit. Let $P_{E, i}$ denote the probability of receiving $H_{1}$ at the central unit when the $i$ th Secondary User sends $H_{0}$ and the probability of receiving $H_{0}$ at the central unit when the $i$ th Secondary User sends $H_{1}$ which can be calculated over $\alpha-\mu$ fading channel as follows:

$$
P_{E, i}=\int_{0}^{\infty} \frac{1}{2} \operatorname{erfc}(\sqrt{\gamma}) \frac{(\alpha / 2) \mu^{\mu} \gamma^{(\alpha \mu / 2)-1}}{\Gamma(\mu) \bar{\gamma}^{\alpha \mu / 2}} e^{-\mu(\gamma / \bar{\gamma})^{\alpha / 2}} d \gamma
$$

where $(1 / 2) \operatorname{erfc}(\sqrt{\gamma})$ is the probability of error for BPSK in an $A W G N$ channel. The shown integral can be put in the form of Laplace transform and hence using [25] (3.7.2-18) and substituting $v=\mu-1, a=\sqrt{\bar{\gamma}(1 / \mu)^{2 / \alpha}}, l / 2 k=1 / \alpha$, the probability of error can be calculated as

$$
\begin{aligned}
& \bar{P}_{E, i}=\frac{\alpha}{4 \Gamma(\mu)} \\
& \times\left[\frac{\sqrt{2} l^{\mu-1 / 2}}{(2 \pi)^{(k+l-1) / 2}} G_{l+1, k+1}^{k+1, l}\left(\left(\frac{\bar{\gamma}(1 / \mu)^{2 / \alpha}}{k}\right)^{k} l^{l} \begin{array}{c}
\Delta(l,-\mu+1), 1 \\
\Delta\left(k, \frac{1}{2}\right), 0
\end{array}\right)\right],
\end{aligned}
$$

where $\Delta(k, a)=a / k,(a+1) / k, \ldots,(a+k-1) / k$.
Consequently, the overall probability of false alarm $Q_{F}$ is obtained as

$$
\begin{aligned}
& Q_{F}=\sum_{i=m}^{N}\left(\begin{array}{c}
N \\
i
\end{array}\right) \operatorname{Prob}\left[\left\{\frac{H_{1}^{\mathrm{CU}}}{H_{0}^{\mathrm{PU}}}\right\}\right]^{i} \\
& \times \operatorname{Prob}\left[\left\{\frac{H_{0}^{\mathrm{CU}}}{H_{0}^{\mathrm{PU}}}\right\}\right]^{N-i}, \\
& Q_{F}=\sum_{i=m}^{N}\left(\begin{array}{c}
N \\
i
\end{array}\right) \operatorname{Prob}\left[\left\{\frac{H_{1}^{\mathrm{CU}}}{H_{0}^{\mathrm{PU}}}\right\}\right]^{i} \\
& \times \operatorname{Prob}\left[1-\left\{\frac{H_{1}^{\mathrm{CU}}}{H_{0}^{\mathrm{PU}}}\right\}\right]^{N-i}, \\
& \operatorname{Prob}\left\{\frac{H_{1}^{\mathrm{CU}}}{H_{0}^{\mathrm{PU}}}\right\} \\
& =\left[\operatorname{Prob}\left\{\frac{H_{1}^{\mathrm{CU}}}{H_{1}^{\mathrm{SU}}}\right\} \times \operatorname{Prob}\left\{\frac{H_{1}^{\mathrm{SU}}}{H_{0}^{\mathrm{PU}}}\right\}\right] \\
& +\operatorname{Prob}\left\{\frac{H_{1}^{\mathrm{CU}}}{H_{0}^{\mathrm{SU}}}\right\} \cdot \operatorname{Prob}\left\{\frac{H_{0}^{\mathrm{SU}}}{H_{0}^{\mathrm{PU}}}\right\} \text {, } \\
& Q_{F}=\sum_{i=m}^{N}\left(\begin{array}{c}
N \\
i
\end{array}\right)\left[\left(1-P_{E, i}\right) P_{F, i}+P_{E, i}\left(1-P_{F, i}\right)\right]^{i} \\
& \times\left[\left(1-P_{E, i}\right)\left(1-P_{F, i}\right)+P_{E, i} P_{F, i}\right]^{N-i},
\end{aligned}
$$

where $H_{1}^{\mathrm{CU}}, H_{1}^{\mathrm{PU}}$, and $H_{1}^{\mathrm{SU}}$ are the hypothesis in the central unit, PU, and SU, respectively. 
Substituting (12) and (30) into (34) yields

$$
\left.\begin{array}{rl}
Q_{F}= & \sum_{i=m}^{N}\left(\begin{array}{c}
N \\
i
\end{array}\right)\left\{\begin{array}{c}
\left(1-\frac{\alpha}{4 \Gamma(\mu)} J(k, l, \alpha, \mu)\right) \frac{\Gamma(m, \lambda / 2)}{\Gamma(m)} \\
+\frac{\alpha}{4 \Gamma(\mu)} J(k, l, \alpha, \mu)\left(1-\frac{\Gamma(m, \lambda / 2)}{\Gamma(m)}\right)
\end{array}\right\} \\
& \times\left\{\left(1-\frac{\alpha}{4 \Gamma(\mu)} J(k, l, \alpha, \mu)\right)\left(1-\frac{\Gamma(m, \lambda / 2)}{\Gamma(m)}\right)\right. \\
& +\frac{\alpha}{4 \Gamma(\mu)} J(k, l, \alpha, \mu) \frac{\Gamma(m, \lambda / 2)}{\Gamma(m)}
\end{array}\right\},
$$

where

$$
\begin{aligned}
J(k, l, \alpha, \mu)= & \frac{\sqrt{2} l^{\mu-1 / 2}}{(2 \pi)^{(k+t-1) / 2}} \\
& \times G_{l+1, k+1}^{k+1, l}\left(\left(\frac{\bar{\gamma}(1 / \mu)^{2 / \alpha}}{k}\right)^{k} l^{l} \begin{array}{c}
\Delta(l,-\mu+1), 1 \\
\Delta\left(k, \frac{1}{2}\right), 0
\end{array}\right) .
\end{aligned}
$$

Similarly, the detection probability $Q_{D}$ can be computed as

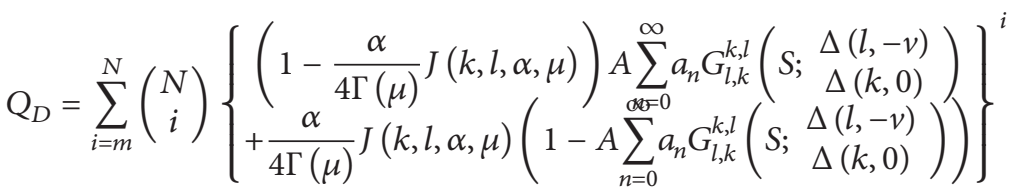

$$
\begin{aligned}
& \times\left\{\begin{array}{c}
\left(1-\frac{\alpha}{4 \Gamma(\mu)} J(k, l, \alpha, \mu)\right)\left(1-A \sum_{n=0}^{\infty} a_{n} G_{l, k}^{k, l}\left(S ; \begin{array}{c}
\Delta(l,-v) \\
\Delta(k, 0)
\end{array}\right)\right) \\
+\frac{\alpha}{8 \Gamma(\mu)} J(k, l, \alpha, \mu) A \sum_{n=0}^{\infty} a_{n} G_{l, k}^{k, l}\left(S ; \begin{array}{c}
\Delta(l,-v) \\
\Delta(k, 0)
\end{array}\right)
\end{array}\right\},
\end{aligned}
$$

where $P_{D, i}$ is the probability of detection of the polarization obtained in (18), (38) for degree detection and axial ratio detection methods, respectively.

Therefore, for AND rule, the overall probability of false alarm and detection are given by

$$
\begin{aligned}
& Q_{F}=\prod_{i=0}^{N}\left\{\begin{array}{c}
\left(1-\frac{\alpha}{4 \Gamma(\mu)} J(k, l, \alpha, \mu)\right) \frac{\Gamma(m, \lambda / 2)}{\Gamma(m)} \\
+\frac{\alpha}{4 \Gamma(\mu)} J(k, l, \alpha, \mu)\left(1-\frac{\Gamma(m, \lambda / 2)}{\Gamma(m)}\right)
\end{array}\right\}, \\
& Q_{D}=\prod_{i=0}^{N}\left\{\begin{array}{c}
\left(1-\frac{\alpha}{4 \Gamma(\mu)} J(k, l, \alpha, \mu)\right) A \sum_{n=0}^{\infty} a_{n} G_{l, k}^{k, l}\left(S ; \begin{array}{c}
\Delta(l,-v) \\
\Delta(k, 0)
\end{array}\right) \\
+\frac{\alpha}{4 \Gamma(\mu)} J(k, l, \alpha, \mu)\left(1-A \sum_{n=0}^{\infty} a_{n} G_{l, k}^{k, l}\left(S ; \begin{array}{c}
\Delta(l,-v) \\
\Delta(k, 0)
\end{array}\right)\right)
\end{array}\right\}^{i} .
\end{aligned}
$$

\section{Cluster-Based Cooperative Spectrum Sensing}

The clustering method is proposed in the cooperative spectrum sensing scheme in order to improve the sensing performance by decreasing the reporting channel error, which is proved to exploit a selection diversity gain [28]. In this paper, we assume that the instantaneous channel state information of the channel is available for the Secondary Users, and the channel between any two Secondary Users in the same cluster is perfect because they are in the vicinity of each other. Figure 3 shows the system model of cluster-based cooperative spectrum sensing. We assume that there are $K$ SUs, who are divided into $L$ clusters. The $i$ th cluster has an integer number $N_{i}$ of SUs, which satisfies

$$
\sum_{i=l}^{L} N_{i}=K .
$$

Firstly, all SUs are assumed to belong to few clusters by the distributed clustering algorithms [29]. Secondly, the Secondary User who has the largest instantaneous reporting channel gain will be selected as the cluster head. Then, the cooperative sensing is carried out through the following steps. Firstly, all SUs perform the local spectrum sensing. Every SU sends a decision to the cluster head. Secondly, the cluster head receives those local decisions from the SUs in the same cluster and then makes the decision according to certain fusion rule. All cluster heads send their decisions to the central unit. In the end, the central unit makes the final decision according to the fusion rule. The cluster head and central unit make the decision according to the OR-rule in order to limit the interference from the SUs to the PU. The $m$-out-of $-N$ fusion rule is adopted in the cluster head and the OR fusion rule is adopted in the central unit [30]. If each cluster has $K / L$ SUs, then the global false alarm probability and detection probability will be given by

$$
\begin{aligned}
& Q_{F}=1 \\
& -\left(\begin{array}{c}
\left.1-\sum_{m=l}^{K / L}\left(\frac{K}{L}\right)\right) \\
\times\left(\frac{\Gamma(m, \lambda / 2)}{\Gamma(m)}\right)^{K}\left(1-\frac{\Gamma(m, \lambda / 2)}{\Gamma(m)}\right)^{K / L-K}
\end{array}\right)^{L},
\end{aligned}
$$




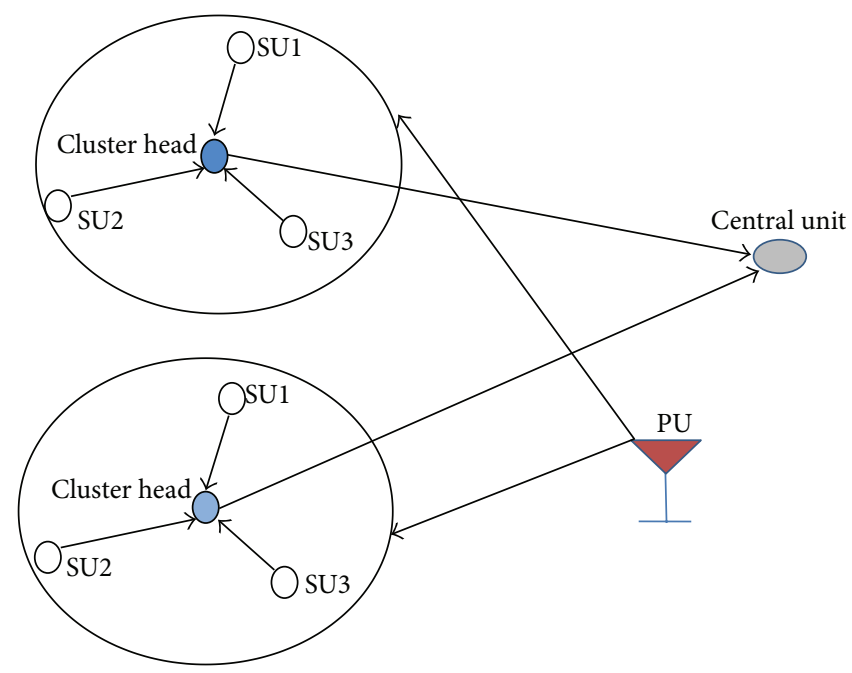

FIgURE 3: Cluster-based spectrum sensing mode.

$Q_{D}=1-\left(1-\sum_{m=l}^{K / L}\left(\underset{m}{\left(\frac{K}{L}\right)}\right)\left(P_{d}\right)^{K}\left(1-p_{d}\right)^{K / L-K}\right)^{L}$

Substituting $P_{d}$ for the degree detection in (18) into (42) yields

$$
Q_{D}=1-\left(\begin{array}{c}
\left.1-\sum_{m=l}^{K / L}\left(\begin{array}{c}
K \\
L
\end{array}\right)\right) \\
\times\left(A \sum_{n=0}^{\infty} a_{n} G_{l, k}^{k, l}\left(S ; \begin{array}{c}
\Delta(l,-v) \\
\Delta(k, 0)
\end{array}\right)\right)^{K} \\
\left(1-A \sum_{n=0}^{\infty} a_{n} G_{l, k}^{k, l}\left(S ; \begin{array}{c}
\Delta(l,-v) \\
\Delta(k, 0)
\end{array}\right)\right)^{K / L-K}
\end{array}\right),
$$

where

$$
\left.\begin{array}{l}
U(v, \mu, z) \\
=\left\{\begin{array}{cc}
A(v, \mu, z) & \alpha=1 \\
\Gamma(v+\mu) U(v, 1-\mu, z) & \alpha=2 \\
\frac{(z / k)^{v} l^{\mu+1 / 2}}{\sqrt{2 \pi}} G_{k+l, k}^{k, k+l} & \\
\times\left[\frac{2}{z^{k}} \mid \Delta(l,-\mu), \Delta(k, v+1)\right. \\
\Delta(k, 0)
\end{array}\right] \alpha \neq 1,2 .
\end{array}\right\},
$$

and substituting $P_{d}$ for the axial ratio detection in (27) into (42) yields

$$
Q_{D}=1-\left(\begin{array}{c}
1-\sum_{m=l}^{K / L}\left(\left(\frac{K}{L}\right)\right) \\
m
\end{array}\left(\begin{array}{c}
1-\sum_{n=0}^{\infty} \frac{\Gamma(N+n)}{\Gamma(n+1) \Gamma(N)} I_{Y_{\mathrm{th}}}\left(n+\frac{1}{2}, N\right) \\
\times \sum_{r=0}^{N}\left(\begin{array}{c}
N \\
r
\end{array}\right)(-1)^{r} U(v, \mu, z)
\end{array}\right)^{K}\right)^{L / L-K}
$$

\section{Results and Discussion}

Results obtained using the proposed methods are presented. Monte Carlo simulation consisting of 100000 independent trials was performed. The degree of freedom is set to 3.5 to make fair comparison between the proposed methods and the energy detection method [31]. The equal and normalized polarization states of the PUs are randomly generated with $\zeta \in(0, \pi / 2), \phi \in(-\pi / 2, \pi / 2)$. It is assumed that SUs use dual polarized antennas, which is necessary to detect the horizontal and vertical components of the wave.

6.1. The Degree of Polarization (DoP) Method. Figure 4 compares the Receiver Operating Characteristic (ROC) of the DoP method with the directional method [18]. It can be noticed from this figure that the DoP method improves the $P_{d}$ at low SNR since the signal is polarized and the noise is unpolarized wave. This makes the signal detection, in particular at low SNR, more efficient, which is the range of interest for CR systems. Therefore, it cannot be used in high SNR but it offers a baseline for comparison.

Figure 5 shows the ROC under AWGN compared with the energy detection (ED) method. It is clear from this figure that the proposed method improves the probability of detection over a wide range of SNR (from $-5 \mathrm{~dB}$ to $5 \mathrm{~dB}$ ). For example, in the case of SNR $=0 \mathrm{~dB}, P_{d}$ improved from $22 \%$ to $88 \%$. Moreover, it is observed that $P_{d}$ at $\mathrm{SNR}=0 \mathrm{~dB}$ for the DoP method is better than $P_{d}$ for the ED method at SNR $=5 \mathrm{~dB}$, meaning the improvement of more than $+5 \mathrm{~dB}$ for the DoP method relative to the ED method. It is also noticed that at $\mathrm{SNR}=10 \mathrm{~dB}$, the DoP method offers $100 \%$ detection probability for all values of $P_{f}$. This improvement is due to the more accurate detection of the polarized portion.

The case of Rayleigh fading channel is considered in Figure 6, which illustrates that fading has a stronger impact on the ED than on the proposed DoP method. This is attributed to the fact that the DoP is based on a second order statistic; that is, the improvement in the probability of 


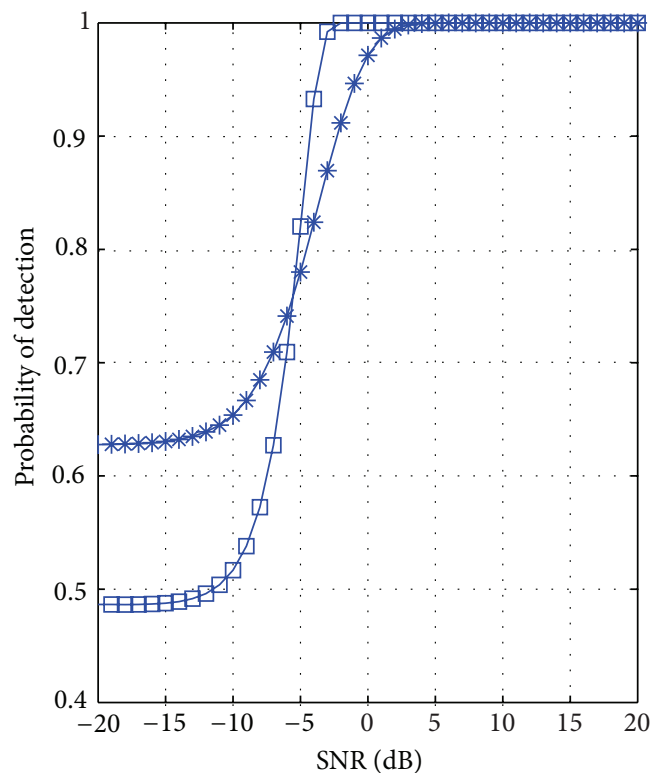

$\square$ AWGN-directional method (Guo et al. 2013) * AWGN-DoP method

FIGURE 4: Probability of detection versus SNR for directional and DoP methods.

detection for SNR $=5 \mathrm{~dB}$ is approximately $30 \%$ at $P_{f}=0.2$. A better performance for the DoP is reported for Nakagami fading as shown in Figure 7, where, at SNR $=0 \mathrm{~dB}$, the improvement is approximately $20 \%$ when the $P_{f}=0.2$. Moreover, the $P_{d}$ at $\mathrm{SNR}=5 \mathrm{~dB}$ for the proposed method is greater than the $P_{d}$ for the ED method at SNR $=10 \mathrm{~dB}$, this means that the improvement is nearly $+7 \mathrm{~dB}$.

It can be noticed that at fading conditions, the depolarization effect increases, which means higher dispersion of polarization states. Therefore, the detection performance decreases. Thus, the smaller the depolarization effect on the primary signal, the more constant the polarization state and thus the better the detection performance.

6.2. The Axial Ratio (AR) Method. To demonstrate the performance of the axial ratio (AR) method, Figure 8 shows the characteristics for different values of $\alpha$ and $\mu$. For $\alpha=2$ and $\mu=2$, the probability of detection is $91 \%$ at $P_{f}=0.2$, which is a superior value relative to the ED method. This can be easily explained since at strong fading less energy is coupled between the cross-polarized channels, which results in small dispersion of the polarization state leading to better detection performance.

Figure 9 shows the superior performance of the AR method relative to the ED method over a wide range of SNR under Rayleigh fading channel. In Figure 10, where Nakagami fading is considered, for $\mathrm{SNR}=-5 \mathrm{~dB}$, an improvement of approximately $60 \%$ in the probability of detection is achieved for $P_{f}=0.2$ and $70 \%$ for $\mathrm{SNR}=5 \mathrm{~dB}$ relative to the ED method. Hence, the AR method is the most robust and applicable detection method in the case of fading condition and/or presence of noise power uncertainty.

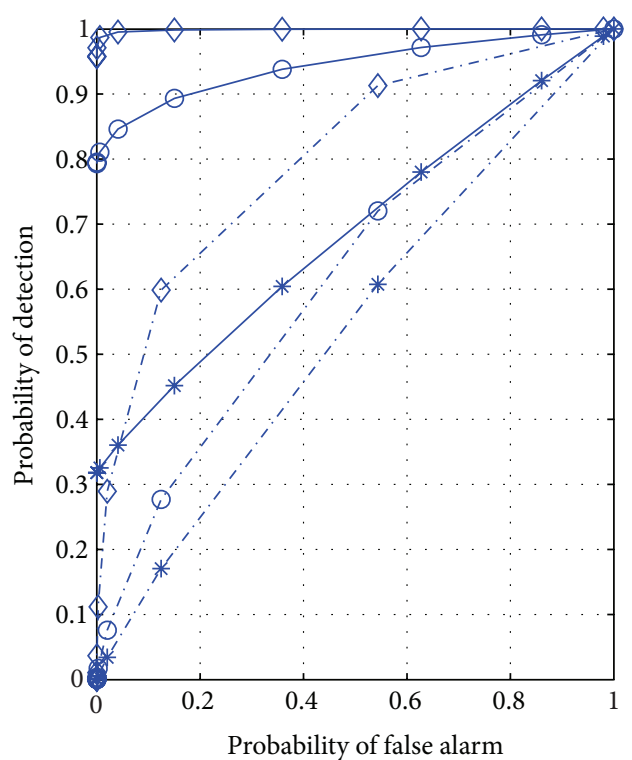

$$
\begin{aligned}
& * \text { DoP method, AWGN, SNR }=-5 \mathrm{~dB} \\
& - \text { DoP method, AWGN, SNR }=0 \mathrm{~dB} \\
& \diamond \text { DoP method, AWGN, SNR }=5 \mathrm{~dB} \\
& *-\text { Energy method, AWGN, SNR }=-5 \mathrm{~dB} \\
& \ominus-\text { Energy method, AWGN, SNR }=0 \mathrm{~dB} \\
& \diamond-\text { Energy method, AWGN, SNR }=5 \mathrm{~dB}
\end{aligned}
$$

FIgURE 5: ROC of the DoP method in AWGN.

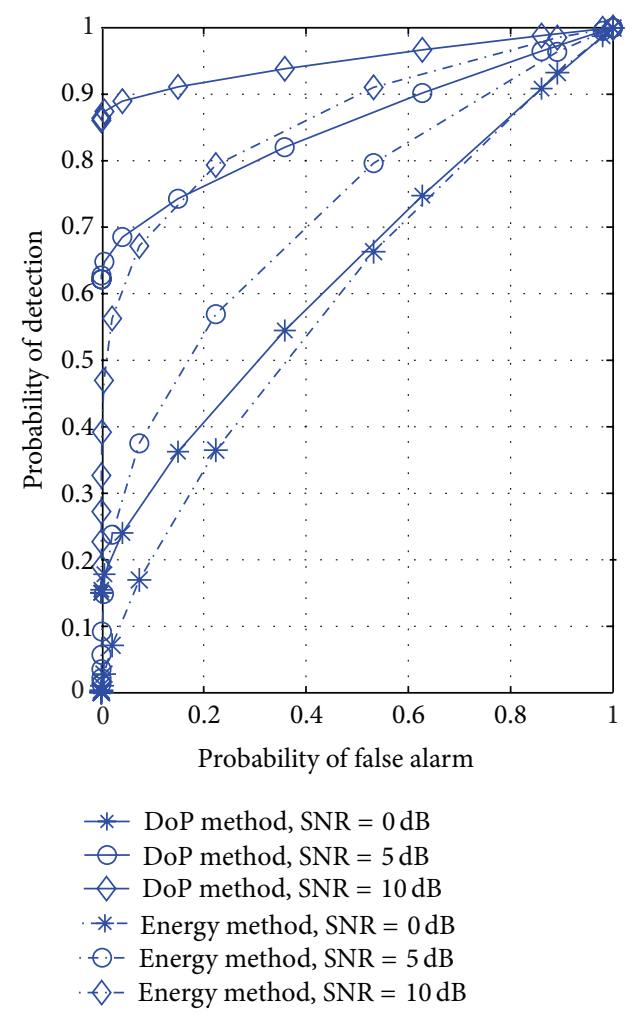

Figure 6: ROC of the DoP method under Rayleigh fading channel. 


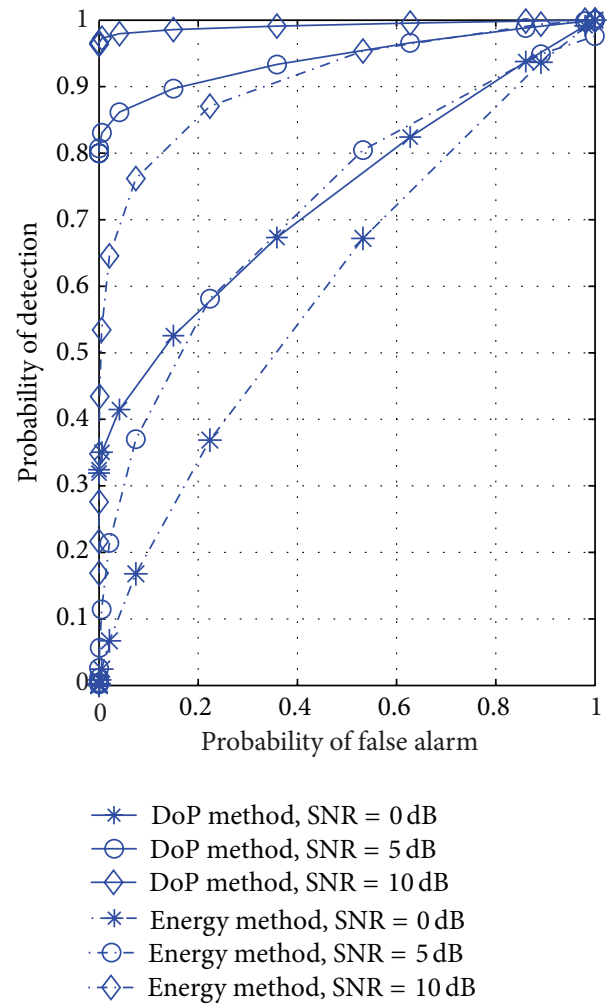

Figure 7: ROC of the DoP method under Nakagami fading channel.

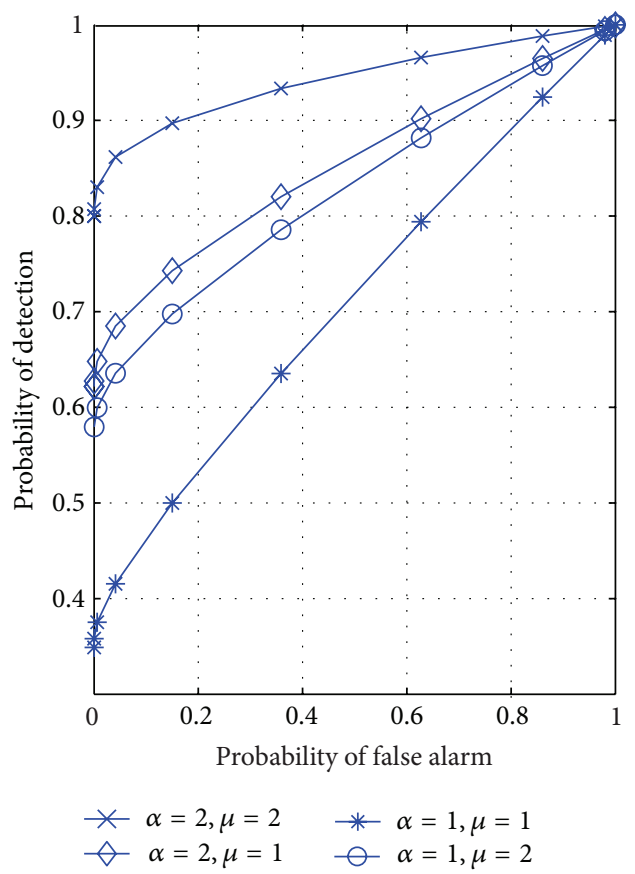

FIgURE 8: ROC of the DoP method under different $\alpha, \mu$ at SNR $=$ $5 \mathrm{~dB}$.

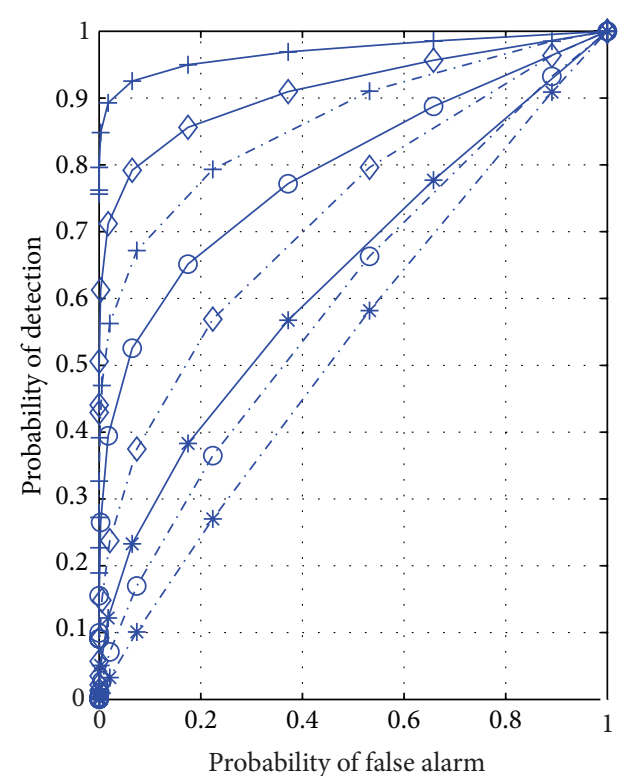

$$
\begin{aligned}
& * \text { AR method, } \mathrm{SNR}=-5 \mathrm{~dB} \\
& -\mathrm{AR} \text { method, } \mathrm{SNR}=0 \mathrm{~dB} \\
& \diamond \mathrm{AR} \text { method, } \mathrm{SNR}=5 \mathrm{~dB} \\
& +\mathrm{AR} \text { method, } \mathrm{SNR}=10 \mathrm{~dB} \\
& *-\text { Energy method, } \mathrm{SNR}=-5 \mathrm{~dB} \\
& \ominus-\text { Energy method, SNR }=0 \mathrm{~dB} \\
& \diamond-\text { Energy method, SNR }=5 \mathrm{~dB} \\
& +- \text { Energy method, SNR }=10 \mathrm{~dB}
\end{aligned}
$$

FIGURE 9: ROC of the AR method under Rayleigh fading channel.

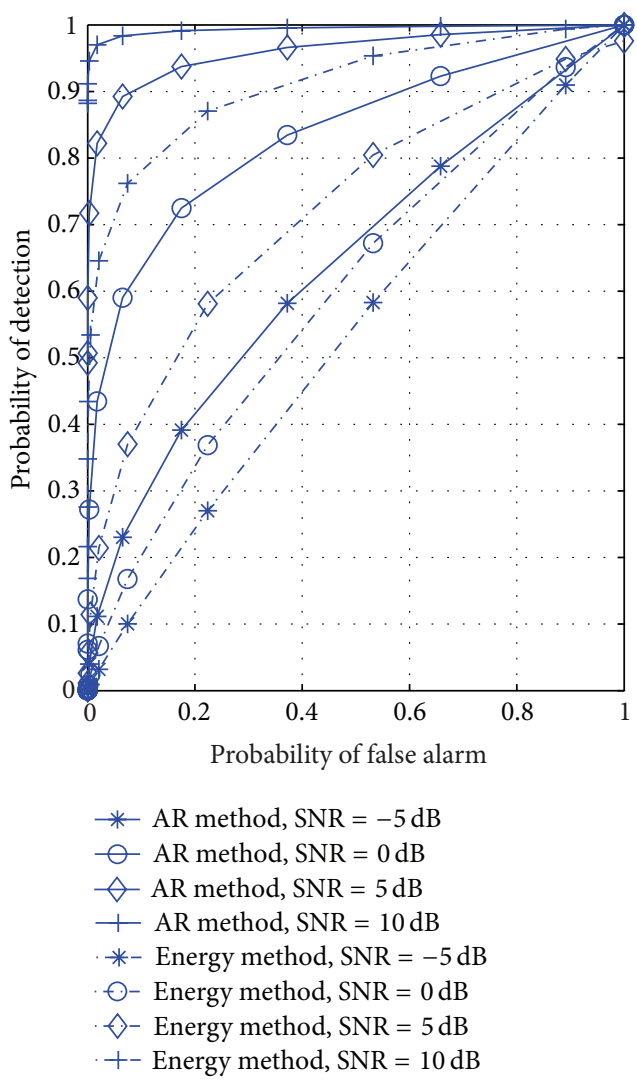

FIGURE 10: ROC of the AR method under Nakagami fading channel. 


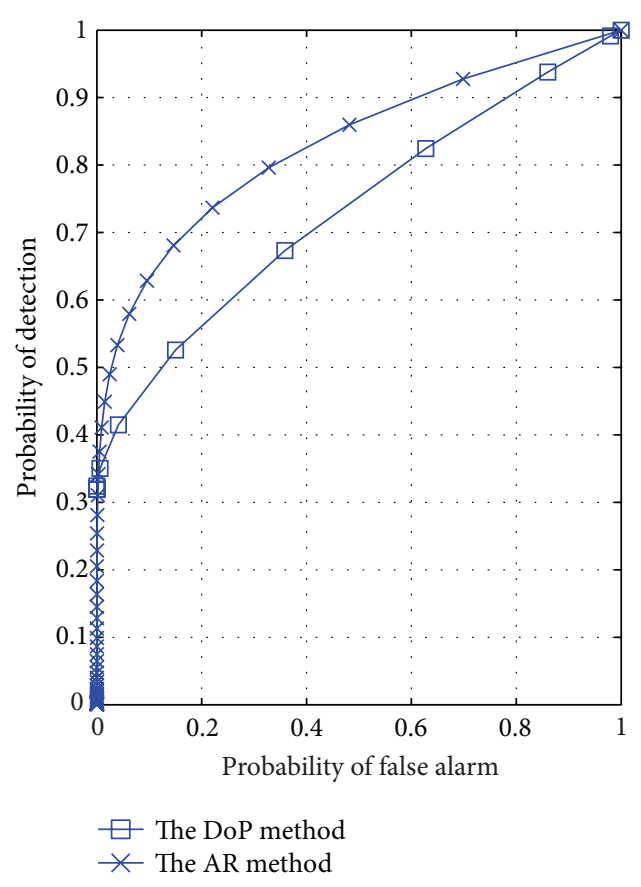

Figure 11: ROC of the two methods (AR, DoP) at $\mathrm{SNR}=0 \mathrm{~dB}$ under Nakagami fading channel.

A comparison between both approaches is drawn in Figure 11 showing the superiority of the AR method relative to the DoP, where for $P_{f}=0.2$, the AR method gives $P_{d}=0.73$ compared to $P_{d}=0.58$ for the DoP. It can be seen that the sensing performance of the AR is better than the DoP method due to the fact that the AR method resists the depolarization effect on the primary signal.

6.3. The Cooperative Scheme. Figure 12 shows the ROC of the cooperative scheme for the DoP method over a wide range of SNR under imperfect Nakagami fading channel. It is clear that the $P_{d}$ is very high at low $P_{f}$ especially at $\mathrm{SNR}=5 \mathrm{~dB}$ and $10 \mathrm{~dB}$. It can be observed that the $P_{d}$ increase rapidly with the increase of the SNR. When the SNR $=10 \mathrm{~dB}$, the $P_{d}$ is almost $100 \%$ for all values of $P_{f}$.

\section{Conclusions}

Spectrum sensing based on Stokes parameters was thoroughly analyzed using new detection statistics, namely, the degree of polarization and the axial ratio. The proposed approaches were studied under different fading scenarios and the obtained results demonstrated superior performance relative to the conventional energy detection method. An extensive study is reported on the two methods and their performance under $\alpha-\mu$ fading channels. The results demonstrated that the proposed algorithms are particularly applicable for the case of unknown primary polarization and/or presence of noise power uncertainty. In general, the proposed methods are better than energy detection in different ranges of the SNR and under different fading conditions. They are,

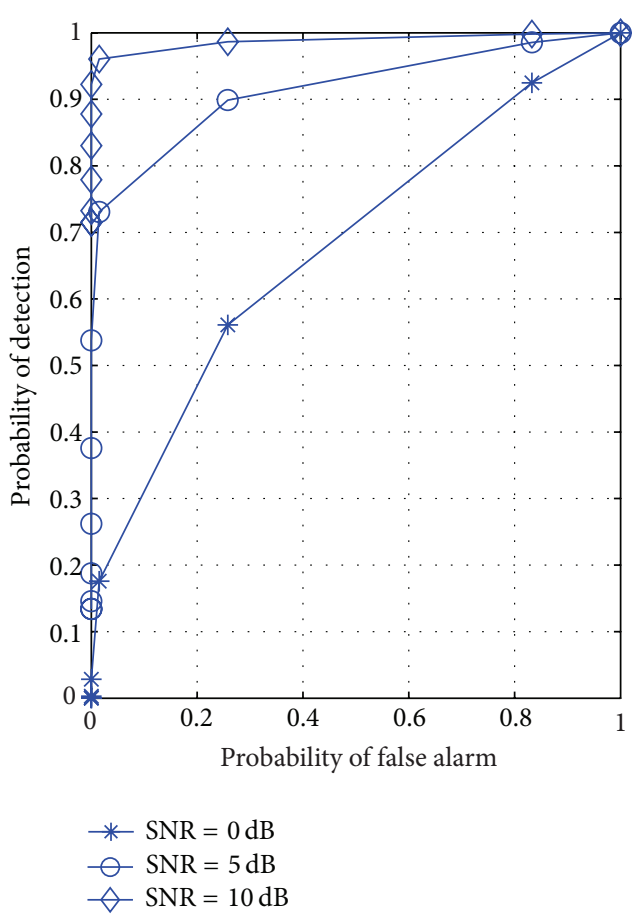

FIGURE 12: ROC of the cooperative scheme of the DoP method under imperfect Nakagami fading channel.

however, more complex in terms of implementation due to the use of two orthogonally polarized antennas. It is also worth noting that the axial ratio method is generally better than the degree of polarization method.

Cooperative spectrum sensing was then considered and shown to be a powerful method for dealing with the hidden terminal problem. Simulation results show that the cluster rule gives superior performance for a wide range of SNR.

\section{Conflict of Interests}

The authors declare that there is no conflict of interests regarding the publication of this paper.

\section{References}

[1] S. Haykin, "Cognitive radio: brain-empowered wireless communications," IEEE Journal on Selected Areas in Communications, vol. 23, no. 2, pp. 201-220, 2005.

[2] A. Sahai and D. Cabric, "Spectrum sensing: fundamental limits and practical challenges," in Proceedings of the IEEE International Symposium on New Frontiers in Dynamic Spectrum Access Network (DySPAN '05), Baltimore, Md, USA, November 2005.

[3] W. Wei, P. Tao, and W. Wenbo, "Optimal power control under interference temperature constraints in cognitive radio network," in Proceedings of the IEEE Wireless Communications and Networking Conference (WCNC '07), pp. 116-120, March 2007.

[4] I. F. Akyildiz, W.-Y. Lee, M. C. Vuran, and S. Mohanty, "A survey on spectrum management in cognitive radio networks," IEEE Communications Magazine, vol. 46, no. 4, pp. 40-48, 2008. 
[5] F. F. Digham, M.-S. Alouini, and M. K. Simon, "On the energy detection of unknown signals over fading channels," IEEE Transactions on Communications, vol. 55, no. 1, pp. 21-24, 2007.

[6] A. V. Dandawate and G. B. Giannakis, "Statistical tests for presence of cyclostationarity," IEEE Transactions on Signal Processing, vol. 42, no. 9, pp. 2355-2369, 1994.

[7] L. Zhang, Y.-C. Liang, Y. Xin, and H. V. Poor, "Robust cognitive beamforming with partial channel state information," IEEE Transactions on Wireless Communications, vol. 8, no. 8, pp. 4143-4153, 2009.

[8] H. Kim, J. Kim, S. Yang, M. Hong, and Y. Shin, "An effective MIMO-OFDM system for IEEE 802.22 WRAN channels," IEEE Transactions on Circuits and Systems II, vol. 55, no. 8, pp. 821$825,2008$.

[9] Z. Quan, S. Cui, A. H. Sayed, and H. V. Poor, "Wideband spectrum sensing in cognitive radio networks," in Proceedings of the IEEE International Conference on Communications (ICC '08), pp. 901-906, May 2008.

[10] Z. Tian, "Compressed wideband sensing in cooperative cognitive radio networks," in Proceedings of the IEEE Global Telecommunications Conference (GLOBECOM '08), pp. 37563760, December 2008.

[11] A. J. Poelman, "On using orthogonally polarized noncoherent receiving channels to detect target echoes in gaussian noise," IEEE Transactions on Aerospace and Electronic Systems, vol. 11, no. 4, pp. 660-663, 1975.

[12] G. M. Vachula and R. M. Barnes, "Polarization detection of a fluctuating radar target," IEEE Transactions on Aerospace and Electronic Systems, vol. 19, no. 2, pp. 250-257, 1983.

[13] R. E. Stovall, "A gaussian noise analysis of the pseudo-coherent discriminant," Tech. Rep. Note 1978-46, M.I.T. Lincoln Laboratory, 1978.

[14] D. P. Meyer and H. A. Mayer, Radar Target Detection: Handbook of Theory and Practice, Academic Press, New York, NY, USA, 1973.

[15] F. Liu, C. Feng, C. Guo, Y. Wang, and D. Wei, "Polarization spectrum sensing scheme for cognitive radios," in Proceedings of the 5th International Conference on Wireless Communications, Networking and Mobile Computing (WiCOM '09), September 2009.

[16] D. Wei, C. Guo, F. Liu, and Z. Zeng, "A SINR improving scheme based on optimal polarization receiving for the cognitive radios," in Proceedings of the IEEE International Conference on Network Infrastructure and Digital Content (IC-NIDC '09), pp. 100-104, November 2009.

[17] F. Liu, C. Feng, C. Guo, Y. Wang, and D. Wei, "Virtual polarization detection: a vector signal sensing method for cognitive radios," in Proceedings of the 71st IEEE Vehicular Technology Conference (VTC-Spring '10), pp. 1-5, Taipei, Taiwan, 2010.

[18] C. Guo, X. Wu, C. Feng, and Z. Zeng, "Spectrum sensing for cognitive radios based on directional statistics of polarization vectors," IEEE Journal on Selected Areas in Communications, vol. 31, no. 3, pp. 379-393, 2013.

[19] K. B. Letaief and W. Zhang, "Cooperative communications for cognitive radio networks," Proceedings of the IEEE, vol. 97, no. 5, pp. 878-893, 2009.

[20] L. P. Murza, "The non coherent polarimetry of noise like radiation," Radio Engineering and Electronic Physics, vol. 23, no. 7, pp. 57-63, 1978.

[21] G. Deschamps, “Techniques for handling elliptically polarized waves with special reference to antennas: part II-geometrical representation of the polarization of a plane electromagnetic wave," Proceedings of the IRE, vol. 39, no. 5, pp. 540-544, 1951.

[22] N. Goodman, "Statistical analysis based on a certain multivariate complex gaussian distribution (an introduction)," Annals of Mathematical Statistics, vol. 34, no. 1, pp. 152-177, 1963.

[23] M. D. Yacoub, “The $\alpha-\mu$ distribution: a physical fading model for the Stacy distribution," IEEE Transactions on Vehicular Technology, vol. 56, no. 1, pp. 27-34, 2007.

[24] M. K. Simon and M. S. Alouini, Digital Communication over Fading Channels, vol. 86, Wiley-IEEE Press, 2004.

[25] A. Prudnikov, Y. A. Brychkov, and O. Marichev, Integrals and Series, vol. 4, Gordon and Breach Science, 1986.

[26] F. W. Olver, D. W. Lozier, R. F. Boisvert, and C. W. Clark, NIST Handbook of Mathematical Functions, Cambridge University Press, 2010.

[27] A. Ghasemi and E. S. Sousa, "Collaborative spectrum sensing for opportunistic access in fading environments," in Proceedings of the 1st IEEE International Symposium on New Frontiers in Dynamic Spectrum Access Networks (DySPAN '05), pp. 131-136, November 2005.

[28] C. Sun, W. Zhang, and K. B. Letaief, "Cluster-based cooperative spectrum sensing in cognitive radio systems," in Proceedings of the IEEE International Conference on Communications (ICC '07), pp. 2511-2515, June 2007.

[29] O. Younis and S. Fahmy, "Distributed clustering in ad-hoc sensor networks: a hybrid, energy-efficient approach," in Proceedings of the 23rd Annual Joint Conference of the IEEE Computer and Communications Societies (INFOCOM '04), vol. 1, pp. 629-640, March 2004.

[30] A. Ghasemi and E. S. Sousa, "Spectrum sensing in cognitive radio networks: the cooperation-processing tradeoff," Wireless Communications and Mobile Computing, vol. 7, no. 9, pp. 10491060, 2007.

[31] J. Ma, G. Zhao, and Y. Li, "Soft combination and detection for cooperative spectrum sensing in cognitive radio networks," IEEE Transactions on Wireless Communications, vol. 7, no. 11, pp. 4502-4507, 2008. 

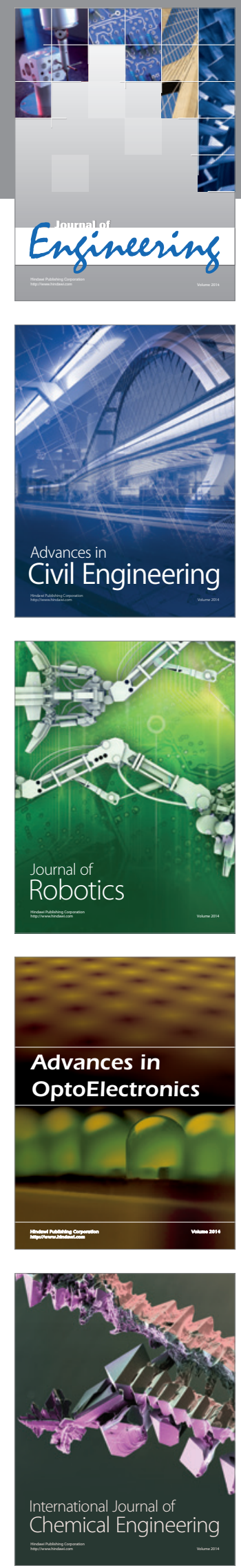

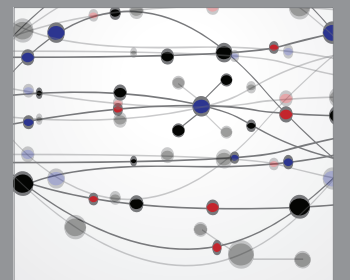

The Scientific World Journal
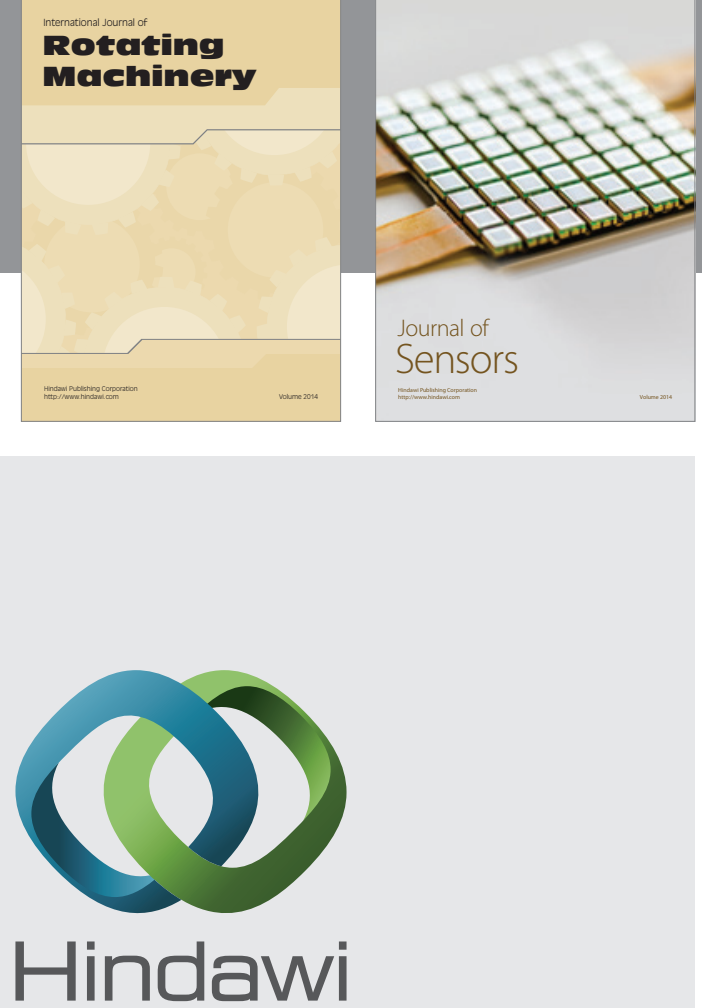

Submit your manuscripts at http://www.hindawi.com
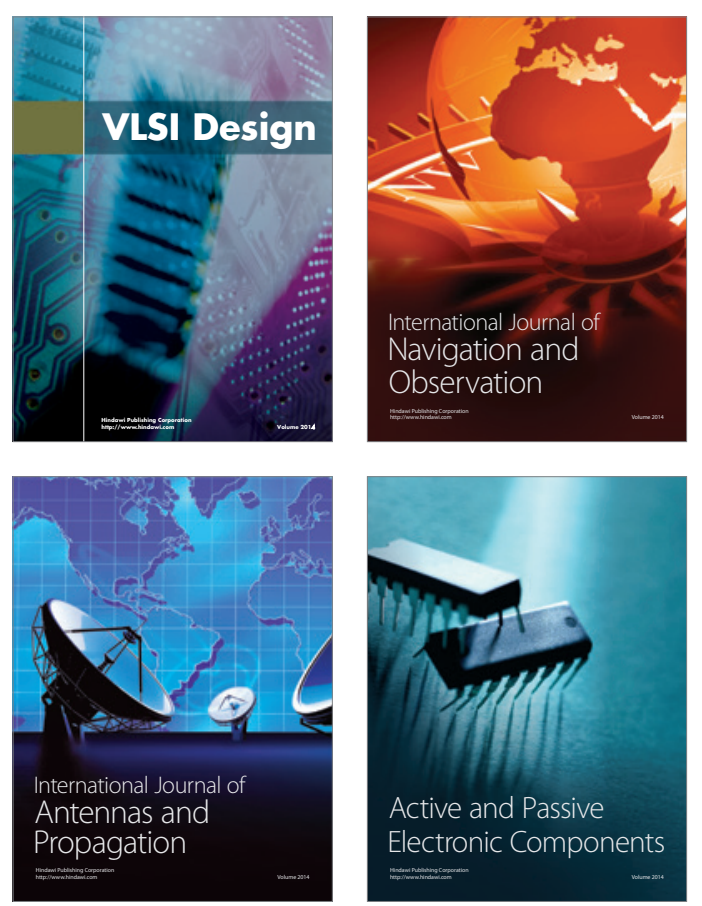
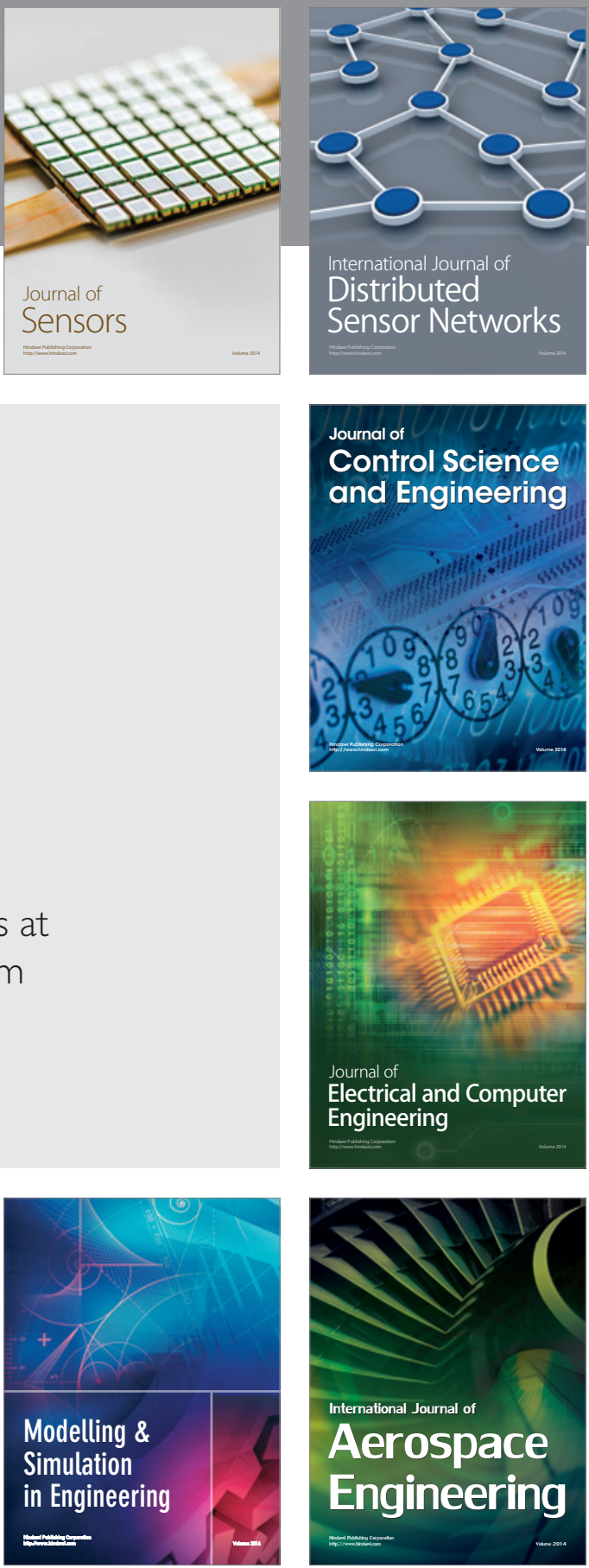

Journal of

Control Science

and Engineering
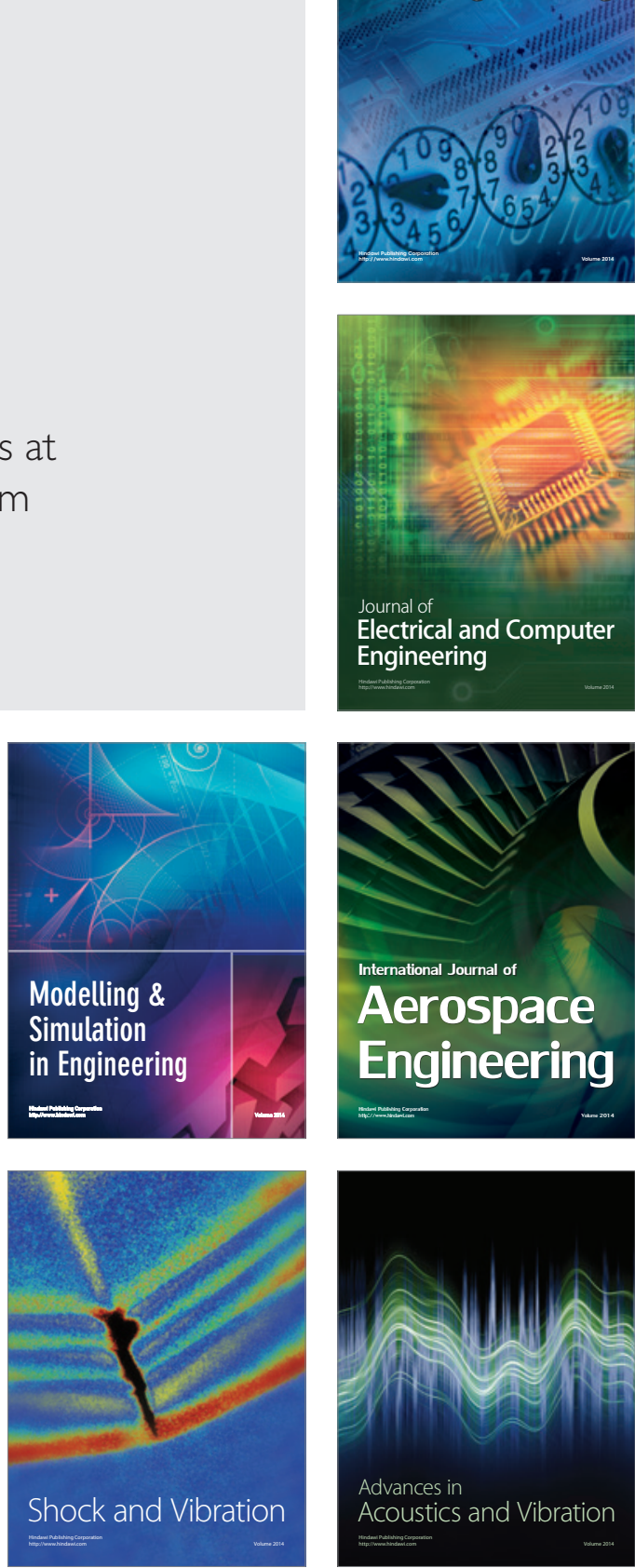